\title{
An acceptance-based intervention for children and adolescents with cancer experiencing acute pain - a single-subject study
}

This article was published in the following Dove Press journal:

Journal of Pain Research

6 September 2017

Number of times this article has been viewed

\author{
Jenny Thorsell Cederberg' \\ JoAnne Dahl ${ }^{2}$ \\ Louise von Essen ${ }^{3}$ \\ Gustaf Ljungman'
}

'Pediatric Oncology, Department of Women's and Children's Health, ${ }^{2}$ Department of Psychology, ${ }^{3}$ Clinical Psychology in Healthcare, Department of Women's and Children's Health, Uppsala University, Uppsala, Sweden
Correspondence: Jenny Thorsell Cederberg

Department of Women's and Children's Health, Uppsala University, The

Children's University Hospital, SE-75I 85 Uppsala, Sweden

$\mathrm{Tel}+46$ I8 6II 0197

$\mathrm{Fax}+46 \quad 18508680$

Email jenny.thorsell.cederberg@kbh.uu.se
Background: Children and adolescents with cancer report pain as one of their most recurrent and troublesome symptoms throughout the cancer trajectory. Pain evokes psychological distress, which in turn has an amplifying effect on the pain experience. Acceptance-based interventions for experimentally induced acute pain predict increased pain tolerance, decreased pain intensity and decreased discomfort of pain. The aim of this study was to preliminarily evaluate an acceptance-based intervention for children and adolescents with cancer experiencing acute pain, with regard to feasibility and effect on pain intensity and discomfort of pain.

Methods: This is a single-subject study with an AB design with a nonconcurrent multiple baseline. Children and adolescents aged four to 18 years undergoing cancer treatment at the Children's University Hospital, Uppsala, Sweden, reporting sustained acute pain were offered participation. Pain intensity and discomfort of pain were measured during baseline and at post-intervention. The intervention consisted of a pain exposure exercise lasting approximately 15 minutes.

Results: Five children participated in the study. All participants completed the intervention and reported that it had helped them to cope with the pain in the moment. All participants reported decreased discomfort of pain at post-measurement, three of whom also reported decreased pain intensity.

Conclusion: The results suggest that an acceptance-based intervention may help children and adolescents with cancer to cope with the pain that is often associated with cancer treatment in spite of pharmacological pain management. The results are tentative but promising and warrant further investigation.

Keywords: acute pain, acceptance, pain intensity, discomfort of pain

\section{Introduction}

Children and adolescents with cancer suffer from multiple physical symptoms, as a result of the cancer itself and/or the cancer treatment, ${ }^{1,2}$ of which pain has been reported as one of the most frequent and distressing ones..$^{3-6}$ Pain naturally evokes anxiety, ${ }^{7}$ and for children with cancer, pain is unsurprisingly associated with psychological distress. ${ }^{8-10}$ The relationship between pain and negative emotions is not unidirectional, but reciprocal, ${ }^{11-13}$ which means that pain not only causes psychological distress, but that psychological distress also amplifies the pain experience. Brain-imaging studies have shown that negative emotions neurologically facilitate pain impulses, while positive emotions inhibit them. ${ }^{14}$ Further, the meaning of pain affects the experienced intensity. ${ }^{15}$ When the pain is perceived as a threat, it is experienced more intensely and its impact on our functioning increases. ${ }^{16}$ Likewise, perceived uncontrollability has the same intensifying effect on the pain experience. ${ }^{17}$ On the other hand, perception 
of control has an inhibitory effect on pain. ${ }^{18}$ In accordance with these findings, psychological interventions incorporating mindfulness and acceptance techniques have been shown to predict increased pain tolerance, decreased pain intensity and decreased discomfort of pain in experimentally induced pain. ${ }^{19-26}$ Mindfulness has been shown to have a mechanistically distinct pain-relief function compared to placebo and sham-mindfulness. ${ }^{27}$ In mindfulness and acceptance interventions, a focused, attentive, nonreactive stance toward unpleasant stimuli is employed, where the aim is to simply observe ongoing internal experiences without further mental evaluation, in contrast to being busy analyzing or avoiding them. ${ }^{28,29}$ This position enables the possibility to choose one's actions instead of rigidly reacting to one's experiences, and at the same time, it attenuates the pain experience. Mindfulness and acceptance have also been shown to be beneficial for persons suffering from chronic pain, ${ }^{30-32}$ and an acceptancebased treatment, Acceptance and Commitment Therapy, has been shown to improve physical as well as psychosocial functioning for both adults and children with chronic pain. ${ }^{33-36}$ In one study investigating acceptance in parents of children experiencing pain during cancer treatment, parents reported that they lacked psychosocial interventions, and that they felt that they were left alone with difficult feelings, during the treatment. ${ }^{37}$ The importance of addressing the psychosocial needs of children with cancer, and their families, in order to reduce their suffering has been emphasized, ${ }^{38}$ and work remains to be done to incorporate psychological treatments into standard cancer care. ${ }^{39,40}$ For instance, psychological interventions could potentially help children with cancer to cope with the pain associated with cancer treatment. This would have an effect on not only their pain experience but also their general well-being. The aim of this study was to preliminarily evaluate an acceptance-based intervention for children and adolescents with cancer experiencing acute pain in spite of pharmacological pain management. This evaluation included investigating preliminary outcomes as well as feasibility with regard to treatment acceptability and implementation. ${ }^{41}$

\section{Methods}

\section{Participants and setting}

Participants were recruited from the pediatric hematology and oncology ward and outpatient clinic at the Children's University Hospital, Uppsala, Sweden. Children between 4 and 18 years of age reporting sustained acute pain were eligible for the study. Insufficient knowledge of Swedish was an exclusion criterion. Participants were identified by the nurses and/or doctors on the ward or in the clinic and informed about the study by a research nurse. Written consent was obtained by the research nurse, from the child if 15 years of age or older and from a parent if under 15 years of age and the child had given its assent. Ten children were invited to participate, one of whom (a 16-year-old boy) declined and nine accepted. Two of the children who accepted to participate, a four-year-old girl and a six-year-old boy, did not want to participate in the measurements and could therefore not be included. For two 15 -year-old girls, the intervention was not possible to perform during their pain episode due to other appointments, and they were consequently excluded. Hence, five participants were included in the study. This is a pilot study of a randomized controlled trial in which the intervention will be evaluated further. The study was approved by the Regional Ethical Review Board in Uppsala, Sweden (Dnr 2012/126). Informed written consent was collected for publication of case details.

\section{Participant A}

Participant A was a seven-year-old boy diagnosed with osteosarcoma in one leg a few months prior to the study. Due to the osteosarcoma, he had been suffering from pain in his knee from approximately one month prior to diagnosis. He had chemotherapy and suffered from mucositis, which is a common side effect of chemotherapy consisting of painful inflammation and ulcers in the mouth and/or gastrointestinal tract. ${ }^{42}$ The primary pain was inflammatory/nociceptive.

\section{Participant B}

Participant B was a 12-year-old boy diagnosed with osteosarcoma in one leg ten months prior to the study. He was treated with chemotherapy for six months. He had been suffering from pain more or less continuously from approximately one month before diagnosis. The source of pain had until one month before the study been mainly tumor-related and postoperative from gastrostomy, appendectomy and tumor surgery applying a complex operative procedure with osteosynthesis material, which had to be expanded on a daily basis, to preserve adequate femoral length. He had a bone-connecting operation one month before the study which resulted in a complicated pain condition including postoperative/nociceptive pain in one leg and neuropathic pain in both legs and feet.

\section{Participant C}

Participant $C$ was a 13-year-old girl with neurofibromatosis and a peripheral malignant nerve sheath tumor in the thorax. Due to the neurofibromatosis, she had had a long history of 
pain primarily in her right arm and shoulder but also in her abdomen and head. Two years prior to the study, her affliction was not considered sufficient to weigh up the risks of surgery, but as her pain increased and she lost sensation and function in her right arm, she had tumor surgery some months before the study. The tumor had malignified, and since parts of the tumor were not possible to remove, chemotherapy was started. Participation in the study was occasioned by postoperative/nociceptive pain resulting from a gastrostomy.

\section{Participant D}

Participant D was a 14-year-old girl with thrombocytopenia and a pain condition of unclear genesis. Thrombocytopenia is not an oncological but a hematological disease. She also had a suspected systemic disease and a suspected low-graded glioma. For a year and a half prior to the study, she had not been feeling well with symptoms such as dizziness, stiffness, pain and fatigue. The pain had worsened over the last year and further intensified during the week prior to the study. The pain was inflammatory/nociceptive with possible neuropathic components.

\section{Participant E}

Participant E was an 18-year-old boy diagnosed with osteosarcoma in one leg with lung metastases, 15 months prior to the study. He had a standard seven-month protocol of chemotherapy which due to delays was prolonged to nine months and followed by five weeks of radiotherapy. He had tumor surgery in his leg, but not in the lungs since all the metastases had disappeared by the time of surgery, eleven months before the study without further complications. After aggravating pain in his left thorax, he was diagnosed with pulmonary metastatic relapse and had surgery to remove the lung metastases. Participation in the study was occasioned by postoperative/nociceptive pain as a result of this surgery.

\section{Pain management}

All children received pharmacological management according to the routines at the unit - meaning treatment with opioids intravenously if there was acute, moderate or severe pain with a possibility to give boluses for break-through pain when required by the patient. In addition to this, all children received paracetamol orally regularly and clonidine intravenously to potentiate the opioids and possibly reduce side effects. The morphine treatment was given as continuous intravenous infusion, and none of the children had received any break-through medication the last hours prior to the psychological intervention. Children with a neuropathic pain component were, in addition, treated with gabapentin orally.

\section{Outcome measures}

Five baseline assessments were performed before the onset of intervention. The measurements included ratings of pain intensity and discomfort of pain. The visual analog scale (VAS) or the Faces Pain Scale-Revised (FPS-R) was used for both outcome variables. The VAS is recommended for children eight years of age and above, while the FPS-R is recommended for younger children (from four years of age). ${ }^{43}$ The VAS is a pain scale consisting of a $100-\mathrm{mm}$ long horizontal line with the anchors "No pain" on the left side and "Worst imaginable pain" on the right side. Respondents are asked to make a mark on the line representing their own level of pain intensity. The mark corresponds to a score, $0-10$, which is shown on the reverse side of the scale. Its responsivity and validity have been supported. ${ }^{44}$ The FPS-R was developed for children ${ }^{45}$ and includes six faces representing different levels of pain intensity, from "No pain" to "Very much pain". Children are asked to select the facial expression that best represents their level of pain. From left to right, the faces are scored $0,2,4,6,8$ and 10 . Interrater agreement is high between children's and pediatric nurses' ratings, ${ }^{46}$ and the FPS-R correlates well with other pain measurements. ${ }^{47}$ Postmeasurements included one assessment for Participants A-D and two assessments for Participant E. Post-measurements were taken immediately after the end of the intervention.

\section{Feasibility}

Feasibility was investigated with regard to treatment acceptability and implementation. To assess treatment acceptability, the proportion of children accepting to participate in the study was calculated. Furthermore, the children's satisfaction with and intention to use the intervention in the future was assessed by questions about their experience of the intervention and whether they would consider using it again when they experienced pain. To assess implementation, the degree to which the children could complete the intervention was calculated. ${ }^{41}$ Additionally, prior to intervention, the children were asked what they typically do to cope with pain.

\section{Intervention}

The intervention was delivered as an addition to the children's standard pain management. It was developed by two psychologists theoretically and clinically familiar with the process of acceptance of pain, tailored for the study population. The intervention consisted of a standardized pain exposure 
exercise, lasting approximately 15 minutes, aiming to help the children to cultivate a nonreactive perspective of painful stimuli. Specifically, the exercise contained instructions on how to practice attentive focus including awareness of their breathing, bodily sensations, thoughts and feelings. Further on, acceptance was instructed through directions to take note of the pain stimuli while simultaneously maintaining an attentive focus, first on their breathing and later also on the painful stimuli themselves. Finally, acceptance was further cultivated by instructions to attend more closely to the physical sensations of the pain stimuli while at the same time practicing a nonreactive stance toward them.

\section{Design and data analysis}

The study had an $\mathrm{AB}$ design with a nonconcurrent multiple baseline. ${ }^{48}$ (In single-subject research, "A" refers to the baseline phase, and " $\mathrm{B}$ " to the intervention phase.) For one participant, the design was expanded to an ABAB design. Primarily, the data was analyzed through visual inspection. The two-standard-deviation-band (2-SD-band) method, which shows the interval of \pm two standard deviations from the mean of the baseline measures, was also applied for data analysis. $^{49}$

\section{Results}

Graphical representations of the ratings of pain intensity and discomfort of pain during baseline and after intervention are presented in Figures 1-5. The 2-SD-band line is presented in each graph. Time points for the baseline measurements

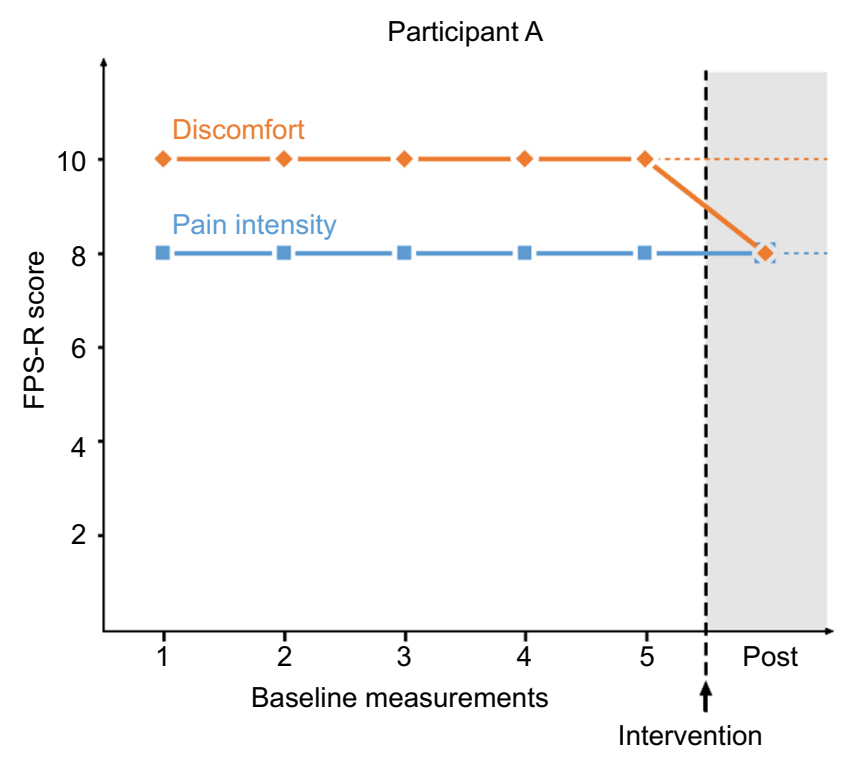

Figure I Participant A's ratings of pain intensity and level of discomfort. Baseline measurements were taken at 53,35, 23, 10 and 0 minutes prior to intervention. Abbreviation: FPS-R, Faces Pain Scale-Revised. are described in each graph. The baselines varied between 25 minutes and 5 hours 10 minutes. Across the participants, there is a change in the pattern of ratings at post-intervention, regardless of length of baseline. For Participant A, there was no variation in the data across baseline measurements, and the standard deviation was therefore " 0 ". Participant E reported no pain after the intervention. Shortly after the end of the intervention, he coughed and vomited, which, due to his very recent lung surgery, caused a quick recurrence of pain. He then expressed a wish to carry out the exercise again. Due to these circumstances, the design was expanded to ABAB for this participant. After the second intervention, he again reported no pain or discomfort.

\section{Feasibility}

One child declined participation in the study. Two children did not want to carry out the assessments. Seven out of ten children thus accepted to participate (two of whom were not able to participate due to practical reasons). Before the start of the intervention, all participants reported examples of avoidant coping strategies exclusively, such as watching TV, playing computer games and trying to think about something else. All the participants in the study completed the intervention. After the intervention, all the participants reported that it had helped them to cope with the pain in the moment. They further expressed that they intended to use the exercise again to cope with pain.

\section{Discussion}

The aim of this study was to preliminarily evaluate an acceptance-based intervention for children and adolescents with cancer experiencing acute pain in spite of pharmacological pain management. The results show that it is feasible to carry out such an intervention for children as young as seven years of age, that the children found the intervention acceptable and that it had helped them to cope with the pain.

Participants A and B reported no decrease in pain intensity but lower levels of discomfort in relation to the pain ratings. This means that for these two participants, their discomfort of pain was alleviated even though the pain intensity remained at the same level. This in line with research on chronic pain as well as studies on experimentally induced pain that show that psychosocial measures improve even when the level of pain is unchanged. ${ }^{35,21}$ For Participants $C$ and D, there was a slight decrease both in reported pain intensity and discomfort of pain. For Participant $C$, the discomfort of pain rating was below the $2-\mathrm{SD}$-band interval indicating that this would not have been expected had the intervention not 


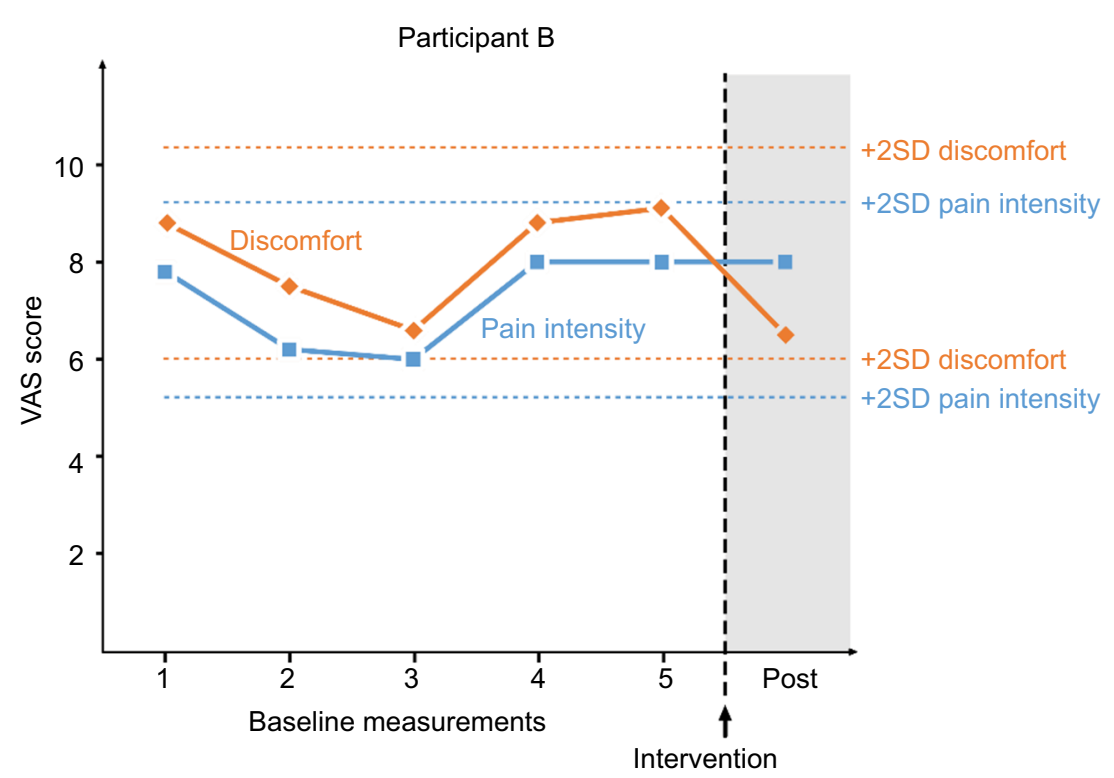

Figure 2 Participant B's ratings of pain intensity and level of discomfort. Baseline measurements were taken at 25, 20, 15,5 and 0 minutes prior to intervention. Abbreviations: VAS, visual analog scale; SD, standard deviation.

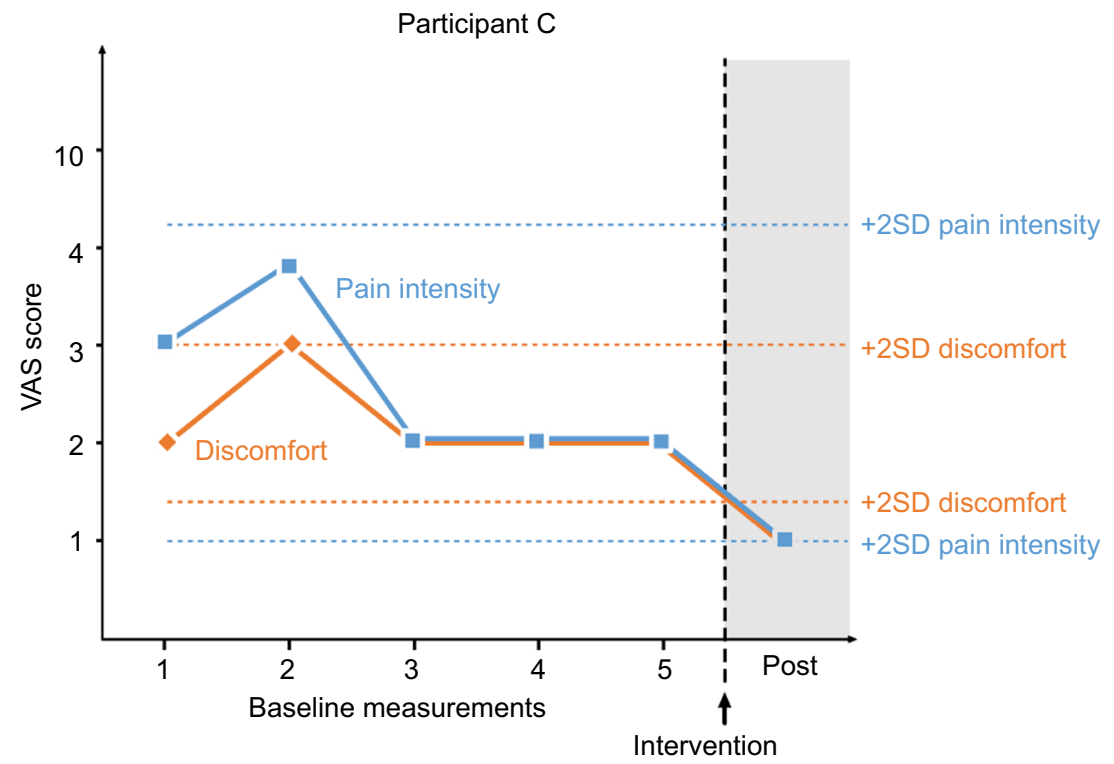

Figure 3 Participant C's ratings of pain intensity and level of discomfort. Baseline measurements were taken at 50, 35, 20, 5 and 0 minutes prior to intervention. Abbreviations: VAS, visual analog scale; SD, standard deviation.

been introduced. For Participant D, both ratings were on the lower 2-SD-band line. This participant expressed that she had some difficulties with the exercise but that she, throughout the exercise, could gradually apply the instructions and that she thought that if the intervention had continued, its effect would have been amplified. This is of course a speculation given the data. For Participant E, both ratings decreased to post-intervention. Following the quick recurrence of pain for this participant due to coughing and vomiting, the intervention was repeated on his request, after which he reported no pain. Due to the particular circumstances in the case of Participant E, an extra post-measurement was implemented after the second post-intervention measurement. The ratings for both outcome variables for all three post-measurements are below the 2-SD-band interval. Previous research on experimentally induced pain showing that acceptance and mindfulness interventions predict both decreased pain intensity and discomfort of pain support these results. ${ }^{19-26}$ Participant E expressed astonishment over the effect of the intervention. The baselines in this study varied between 25 minutes to just 
over five hours, and a change in the response pattern is seen at post-intervention across the participants regardless of differing lengths of baselines. Three of the children who were invited to participate in the study declined. A male adolescent declined when he was informed of the study. It is difficult to say whether he did not want to participate in the study per se or in the intervention. Two young children, four and six years of age, accepted to participate but were then reluctant to perform the measurements and were excluded from the study. The children were nevertheless offered the intervention

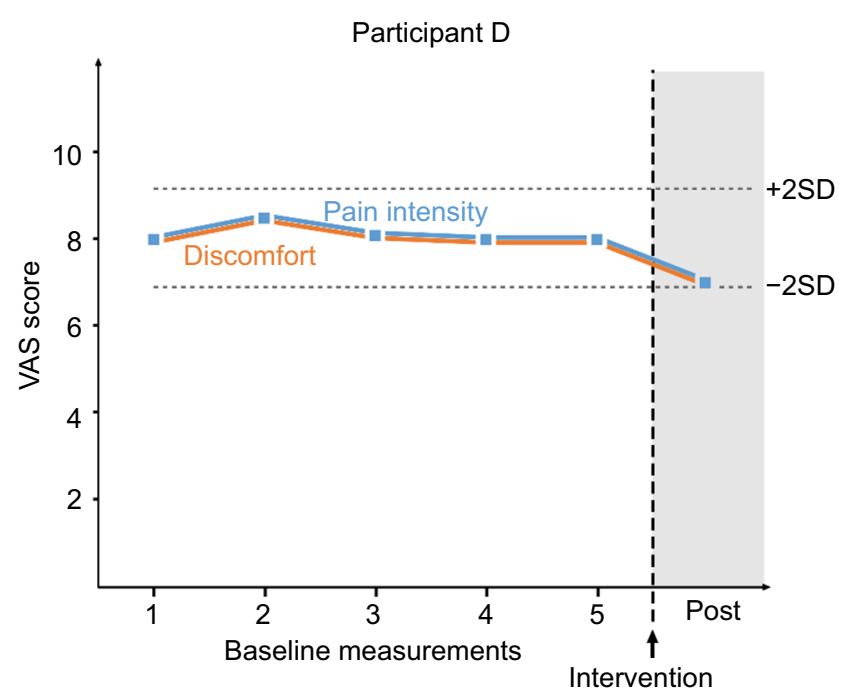

Figure 4 Participant D's ratings of pain intensity and level of discomfort. Baseline measurements were taken at $25,15,10,5$ and 0 minutes prior to intervention. Abbreviations: VAS, visual analog scale; SD, standard deviation. but declined. It is therefore reasonable to conclude that not all children are willing to take part in the intervention. Seven out of ten children accepted to participate in the study, and all of the five participants who started the intervention completed it. Note that all the children reported only avoidant coping strategies before onset of the intervention. Hence, they reported no previous experience of acceptance-based coping for pain and could still both complete and benefit from the intervention. Although possibly axiomatic, it should be mentioned that the intervention is not to be seen as a substitute for pharmacological treatment, but rather as a complement to the children's pain-coping repertoire. The effect of the intervention was most noticeable for Participant E, who was the oldest participant, 18 years of age. At the same time, the effect of the intervention was least evident for the youngest participant, Participant A, who was seven years of age. This is presumably not a coincidence, given that the participant needs to understand the instructions of the intervention and to apply them. This requires rather advanced cognitive abilities, impulse control and emotion regulation, all of which develop throughout childhood and adolescence and may obviously be more developed for an older adolescent than a young child. Nevertheless, the fact that Participant A could carry out the exercise and reported less discomfort by the end of the intervention shows that it may be helpful even for younger children. Another possible moderating factor may be level of pain intensity at baseline. Three of the participants, Participants A, B and D, reported

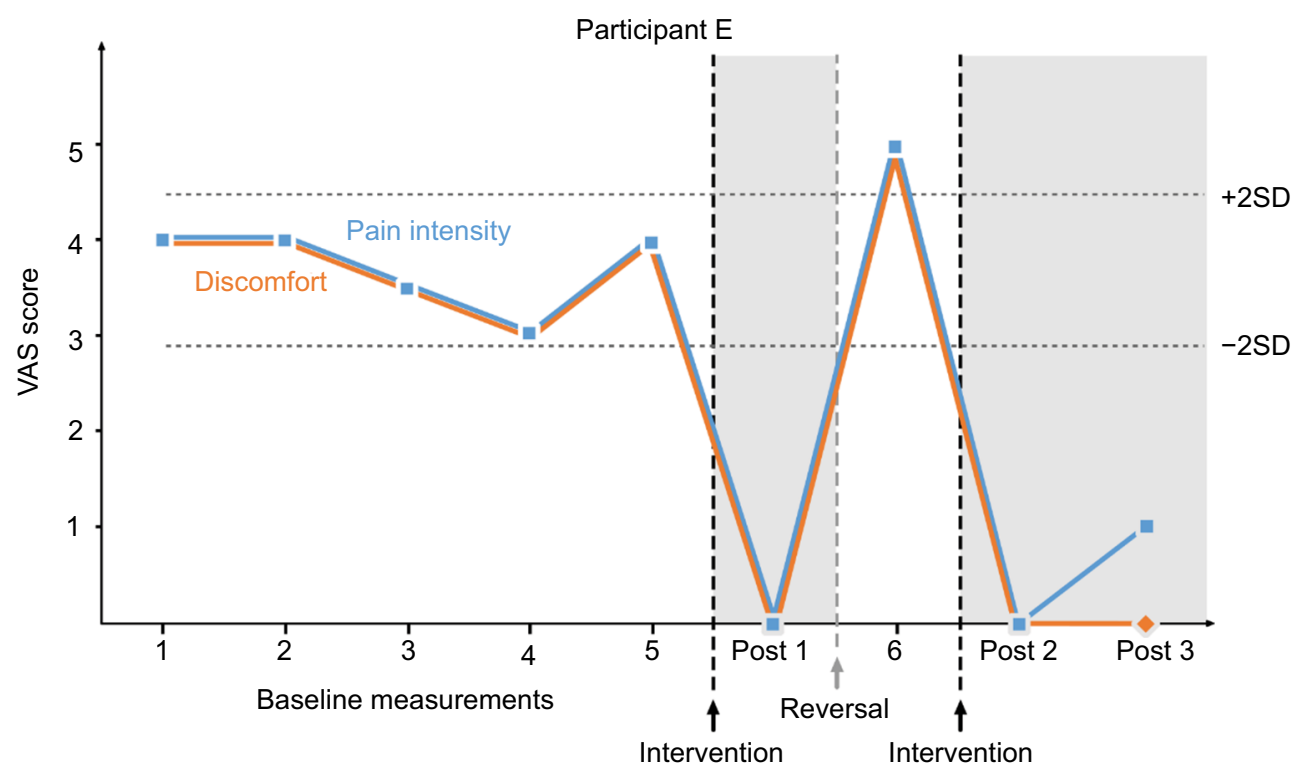

Figure 5 Participant E's ratings of pain intensity and level of discomfort. Baseline measurements were taken at 5 hours 10 minutes, 2 hours 20 minutes, I hour 20 minutes, 5 minutes and 0 minutes prior to intervention. Post 3 was taken 5 minutes after Post 2.

Abbreviations: VAS, visual analog scale; SD, standard deviation. 
high pain intensity at baseline. For two of them, pain intensity was unchanged, while discomfort of pain was reduced. At the same time, Participants $\mathrm{C}$ and $\mathrm{E}$ reported lower pain intensity at baseline and reduced pain intensity at post-measurement. This may suggest that the intervention would be most beneficial for persons with lower baseline pain. On the other hand, pain intensity was reduced as well as discomfort of pain for Participant D. In accordance with her speculation of a more pronounced effect had the intervention been prolonged, it may also be that persons reporting higher baseline pain intensity need longer interventions with more time to practice. This is an empirical question yet to be unfolded.

\section{Study limitations}

The study had an $\mathrm{AB}$ design with a nonconcurrent multiple baseline. An ABAB design would have been preferable, if applicable, to improve internal validity. Given the nature of the intervention, a reversal of the effect was, however, not considered probable after withdrawal of the intervention, and the $\mathrm{ABAB}$ design is not appropriate for evaluating interventions that are difficult to reverse,${ }^{50}$ such as in behavioral, as opposed to medical, interventions. For Participant E, a reversal of the effect was, nevertheless, evident shortly after the withdrawal of the intervention due to him coughing and vomiting after very recently having had lung surgery. For this participant, the design was therefore extended to an $\mathrm{ABAB}$ design. For the remaining participants, this was not considered to be reasonable. A nonconcurrent multiple-baseline design where an effect of an intervention is seen regardless of the length of the baseline also improves the internal validity of a study. Further, more than one post-intervention measurement is normally desirable in single-subject studies. In view of the fact that a hospitalized pediatric oncology patient has numerous medical and other appointments over the course of one day, it would have been difficult to measure a possible prolonged effect of the intervention reliably since the data would potentially be affected by many confounding variables. For a pediatric oncology patient, a normal day in the hospital includes assessments, tests, check-ups and treatment appointments by/with assistant nurses, nurses, doctors, physiotherapists, dietitians, welfare officers, psychologists, hospital school teachers and so forth. Apart from all care-related appointments, they need time to attend to their personal hygiene, meals and social meetings. Further, post-intervention measurements were therefore not included since they were considered to be at risk of being afflicted with excessive noise in the data. A controlled trial would be more appropriate to investigate prolonged effects of the intervention. The analytical approach in the study was mainly visual inspection of the data, which is the foundation of analysis in single-subject research. ${ }^{49}$ The 2-SD-band method was also applied. This method was not ideal for the study as it assumes that data is normally distributed and that there are several post-intervention data points. Bearing this in mind, the method still provides an indication of the interval that the post-data point would likely fall within had the intervention not been introduced. Other methods to accompany the visual analysis in single-subject research are, for example, the celeration line, the $C$-statistic, ${ }^{49}$ the percentage of nonoverlapping data ${ }^{51}$ and the nonoverlap of all pairs method. ${ }^{52}$ These were not applicable on account of too few data points.

\section{Generalizability}

Both sexes and children from the age of seven to 18 years were represented in the study, and the participants reported different kinds of pain, including postoperative and inflammatory nociceptive as well as neuropathic pain. Furthermore, emotional and cognitive factors have been shown to neurologically both facilitate and inhibit pain impulses. Mindfulness and acceptance-based interventions have been shown to attenuate the pain experience, by influencing these emotional and cognitive processes. The intervention of the study is hence trans-diagnostic and theoretically applicable to any type of pain. This supports the generalizability of the results of the study. However, given that the study is a pilot study of five participants with a single-subject research design, the results can still not be considered generalizable, which would require, if not a randomized controlled then at least, a controlled trial.

\section{Conclusion}

In summary, this is a single subject-study investigating feasibility and preliminary effect on pain intensity and discomfort of pain of an acceptance-based intervention for children and adolescents experiencing acute pain during cancer treatment in spite of pharmacological pain management. The results are highly tentative but promising and warrant further investigation.

\section{Acknowledgments}

This study was supported by grants from the Swedish Childhood Cancer Foundation (FTJH11/002 and PR2013/0058) and the Swedish Cancer Society (CAN2013/749). The authors would like to thank the research nurses of the pediatric oncology center in Uppsala, Sweden, Maria Flink, 
Susanne Preinitz Lindberg and Katarina Wallin, for their assistance in information dissemination, recruitment and measurement assessment in the study.

\section{Disclosure}

The authors report no conflicts of interest in this work.

\section{References}

1. Baggott C, Dodd M, Kennedy C, Marina N, Miaskowski C. Multiple symptoms in pediatric oncology patients: a systematic review. J Pediatr Oncol Nurs. 2009;26(6):325-339.

2. Miller E, Jacob E, Hockenberry MJ. Nausea, pain, fatigue, and multiple symptoms in hospitalized children with cancer. Oncol Nurs Forum. 2011;38(5):E382-E393.

3. Ljungman G, Kreuger A, Gordh T, Berg T, Sörensen S, Rawal N. Treatment of pain in pediatric oncology: a Swedish nationwide survey. Pain. 1996;68(2-3):385-394.

4. Yeh CH, Chiang YC, Chien LC, Lin L, Yang CP, Chuang HL. Symptom clustering in older Taiwanese children with cancer. Oncol Nurs Forum. 2008;35(2):273-281.

5. Twycross A, Parker R, Williams A, Gibson F. Cancer-related pain and pain management: sources, prevalence, and the experiences of children and parents. J Pediatr Oncol Nurs. 2015;32(6):369-384.

6. International Association for the Study of Pain (IASP). 2008-2009 global year against cancer pain. Available from: http://www.iasp-pain. org/GlobalYear/CancerPain. Accessed March 8, 2017.

7. Linton SJ, Shaw WS. Impact of psychological factors in the experience of pain. Phys Ther. 2011;91(5):700-711.

8. Hedström M, Haglund K, Skolin I, von Essen L. Distressing events for children and adolescents with cancer: child, parent, and nurse perceptions. J Pediatr Oncol Nurs. 2003;20(3):120-132.

9. Pöder U, Ljungman G, von Essen L. Parents' perceptions of their children's cancer-related symptoms during treatment: a prospective, longitudinal study. J Pain Symptom Manage. 2010;40(5):661-670.

10. Hedén L, Pöder U, von Essen L, Ljungman G. Parents' perceptions of their child's symptom burden during and after cancer treatment. $J$ Pain Symptom Manage. 2013;46(3):366-375.

11. Wiech K, Ploner M, Tracey I. Neurocognitive aspects of pain perception. Trends Cogn Sci. 2008;12(8):306-313.

12. Wiech K, Tracey I. The influence of negative emotions on pain: behavioral effects and neural mechanisms. Neuroimage. 2009;47(3):987-994.

13. Tracey I. Getting the pain you expect: mechanisms of placebo, nocebo and reappraisal effects in humans. Nat Med. 2010;16(11):1277-1283.

14. Denk F, McMahon SB, Tracey I. Pain vulnerability: a neurobiological perspective. Nat Neurosci. 2014;17(2):192-200.

15. Arntz A, Claassens L. The meaning of pain influences its experienced intensity. Pain. 2004;109(1-2):20-25.

16. Zale EL, Lange KL, Fields SA, Ditre JW. The relation between pain-related fear and disability: a meta-analysis. J Pain. 2013;14(10):1019-1030.

17. Bräscher AK, Becker S, Hoeppli ME, Schweinhardt P. Different brain circuitries mediating controllable and uncontrollable pain. J Neurosci. 2016;36(18):5013-5025.

18. Salomons TV, Johnstone T, Backonja MM, Davidson RJ. Perceived controllability modulates the neural response to pain. $J$ Neurosci. 2004;24(32):7199-7203.

19. Kakigi R, Nakata $H$, Inui $K$, et al. Intracerebral pain processing in a Yoga Master who claims not to feel pain during meditation. Eur J Pain. 2005;9(5):581-589.

20. Grant JA, Rainville P. Pain sensitivity and analgesic effects of mindful states in Zen meditators: a cross-sectional study. Psychosom Med. 2009;71(1):106-114.

21. Perlman DM, Salomons TV, Davidson RJ, Lutz A. Differential effects on pain intensity and unpleasantness of two meditation practices. Emotion. 2010;10(1):65-71.
22. Brown CA, Jones AK. Meditation experience predicts less negative appraisal of pain: electrophysiological evidence for the involvement of anticipatory neural responses. Pain. 2011;150(3):428-438.

23. Zeidan F, Gordon NS, Merchant J, Goolkasian P. The effects of brief mindfulness meditation training on experimentally induced pain. J Pain. 2010;11(3):199-209.

24. Zeidan F, Martucci KT, Kraft RA, Gordon NS, McHaffie JG, Coghill $\mathrm{RC}$. Brain mechanisms supporting the modulation of pain by mindfulness meditation. J Neurosci. 2011;31(14):5540-5448.

25. Choi KE, Rampp T, Saha FJ, Dobos GJ, Musial F. Pain modulation by meditation and electroacupuncture in experimental submaximum effort tourniquet technique (SETT). Explore. 2011;7(4):239-245.

26. Forsyth L, Hayes LL. The effects of acceptance of thoughts, mindful awareness of breathing, and spontaneous coping on an experimentally induced pain task. Psychol Rec. 2014;64(3):447-455.

27. Zeidan F, Emerson NM, Farris SR, et al. Mindfulness meditationbased pain relief employs different neural mechanisms than placebo and sham mindfulness meditation-induced analgesia. $J$ Neurosci. 2015;35(46):15307-15325.

28. Keogh E, Bond FW, Hanmer R, Tilston J. Comparing acceptance- and control-based coping instructions on the cold-pressor pain experiences of healthy men and women. Eur J Pain. 2005;9(5):591-598.

29. Buhle J, Wager TD. Does meditation training lead to enduring changes in the anticipation and experience of pain? Pain. 2010;150(3): 382-383.

30. McCracken LM, Gutiérrez-Martínez O. Processes of change in psychological flexibility in an interdisciplinary group-based treatment for chronic pain based on Acceptance and Commitment Therapy. Behav Res Ther. 2011;49(4):267-274.

31. Vowles KE, Witkiewitz K, Sowden G, Ashworth J. Acceptance and commitment therapy for chronic pain: evidence of mediation and clinically significant change following an abbreviated interdisciplinary program of rehabilitation. J Pain. 2014;15(1):101-113.

32. Cederberg JT, Cernvall M, Dahl J, von Essen L, Ljungman G. Acceptance as a mediator for change in acceptance and commitment therapy for persons with chronic pain? Int J Behav Med. 2016;23(1): $21-29$.

33. American Psychological Association (APA). Evidence of psychological treatments. Available from: http://www.div12.org/psychological-treatments/disorders/chronic-or-persistent-pain/acceptance-and-commitment-therapy-for-chronic-pain/. Accessed March 8, 2017.

34. Veehof MM, Oskam MJ, Schreurs KM, Bohlmeijer ET. Acceptancebased interventions for the treatment of chronic pain: a systematic review and meta-analysis. Pain. 2011;152(3):533-542.

35. Hughes LS, Clark J, Colclough JA, Dale E, McMillan D. Acceptance and commitment therapy (ACT) for chronic pain: a systematic review and meta-analyses. Clin J Pain. 2017;33(6):552-568.

36. Weiss KE, Hahn A, Wallace DP, Biggs B, Bruce BK, Harrison TE. Acceptance of pain: associations with depression, catastrophizing, and functional disability among children and adolescents in an interdisciplinary chronic pain rehabilitation program. $J$ Pediatr Psychol. 2013;38(7):756-765.

37. Thorsell Cederberg J, Weineland Strandskov S, Dahl J, Ljungman G. Parents' relationship to pain during children's cancer treatment - a preliminary validation of the Pain Flexibility Scale for parents. J Pain Res. 2017;10:507-514.

38. Wiener L, Viola A, Koretski J, Perper ED, Patenaude AF. Pediatric psycho-oncology care: standards, guidelines, and consensus reports. Psychooncology. 2015;24(2):204-211.

39. International Society of Paediatric Oncology (SIOP). Psycho-social Task Force. Available from: http://siop-online.org/psycho-social-taskforce-2/. Accessed March 9, 2017.

40. International Society of Paediatric Oncology (SIOP). Pediatric PsychoOncology (PPO). Available from: http://siop-online.org/pediatricpsycho-oncology-ppo-2/. Accessed March 9, 2017.

41. Bowen DJ, Kreuter M, Spring B, et al. How we design feasibility studies. Am J Prev Med. 2009;36(5):452-457. 
42. Miller MM, Donald DV, Hagemann TM. Prevention and treatment of oral mucositis in children with cancer. J Pediatr Pharmacol Ther. 2012;17(4):340-350.

43. McGrath PJ, Walco GA, Turk DC, et al; PedIMMPACT. Core outcome domains and measures for pediatric acute and chronic/recurrent pain clinical trials: PedIMMPACT recommendations. J Pain. 2008;9(9):771-783.

44. Ferreira-Valente MA, Pais-Ribeiro JL, Jensen MP. Validity of four pain intensity rating scales. Pain. 2011;152(10):2399-2404.

45. Bieri D, Reeve RA, Champion GD, Addicoat L, Ziegler JB. The faces pain scale for the self-assessment of the severity of pain experienced by children: development, initial validation, and preliminary investigation for ratio scale properties. Pain. 1990;41(2):139-150.

46. Goodenough B, Addicoat L, Champion GD, et al. Pain in 4- to 6-year-old children receiving intramuscular injections: a comparison of the faces pain scale with other self-report and behavioral measures. Clin J Pain. 1997;13(1):60-73.
47. Hicks CL, von Baeyer CL, Spafford PA, van Korlaar I, Goodenough B. The Faces Pain Scale - Revised: toward a common metric in pediatric pain measurement. Pain. 2001;93(2):173-183.

48. Alnahdi GH. Single-subject designs in special education: advantages and limitations. J Res Spec Educ Needs. 2015;15(4):257-265.

49. Backman CL, Harris SR. Case studies, single-subject research and N of 1 randomized trials: comparisons and contrasts. Am J Phys Med Rehabil. 1999;78(2):170-176.

50. Gast DL. Single Subject Research Methodology in Behavioral Sciences. New York: Routledge; 2010.

51. Scruggs TE, Mastropieri MA. PND at 25: past, present, and future trends in summarizing single-subject research. Remedial Spec Educ. 2013;34(1):9-19.

52. Parker RI, Vannest K. An improved effect size for single-case research: nonoverlap of all pairs. Behav Ther. 2009;40(4):357-367.
Journal of Pain Research

\section{Publish your work in this journal}

The Journal of Pain Research is an international, peer reviewed, open access, online journal that welcomes laboratory and clinical findings in the fields of pain research and the prevention and management of pain. Original research, reviews, symposium reports, hypothesis formation and commentaries are all considered for publication.

\section{Dovepress}

The manuscript management system is completely online and includes a very quick and fair peer-review system, which is all easy to use. Visit http://www.dovepress.com/testimonials.php to read real quotes from published authors. 